\section{Estimation of Tomato Bruising by Mechanical Impact Force Using Multivariate Analysis}

\author{
Jinshi Cui, Myongkyoon Yang, Daesik Son, Seongmin Park, \\ and Seong-In Cho ${ }^{1}$
}

Department of Biosystems and Biomaterials Science, Seoul National University, 1 Gwanak-ro, Gwanak-gu, Seoul 08826, Republic of Korea

Additional index words. fruit quality, mechanical impact, postharvest, mathematic models, storage, susceptibility

\begin{abstract}
Fresh fruit bruising caused by mechanical impact is the most problematic effect of mechanical postharvest processing of tomatoes. The conventional postharvest process of tomato fruit (Solanum lycopersicum L.) handling was surveyed from harvest to shipping preparation at a farm in Toechon in the Republic of Korea. Acceleration sensors located on the harvest boxes and fruit skin showed that the most severe impact forces $(>10 \mathrm{~g})$ occurred while fruit was moved in and out of the harvest box. Next, multivariate analysis was used to evaluate nondestructively the susceptibility of tomatoes to bruising by developing five estimation models incorporating quality factors and the presence of bruising resulting from peak contact forces. Linear regression models, artificial neural network (ANN) regression models, and a logistic regression model were built; the primary dependent variables were the rates of weight loss and firmness loss, and impactinduced bruising. The impact force was controlled by using a pendulum, which produced four levels of impact force on tomato fruit. Increasing the relative humidity (RH) and firmness, and decreasing the temperature and degree of weight loss decreased tomato fruit bruise damage. In addition, the proposed drop-impact measurement technique, with multivariate analysis, can be used to evaluate tomato quality nondestructively.
\end{abstract}

Consumers favor high-quality fruits, which are evaluated on their taste and appearance. For this reason, the fruits supplied to markets should meet the international quality standards for firmness, freshness, and certain other quality parameters (Lubes and Goodarzi, 2017). During the postharvest process, horticultural products are subjected to various external forces. When these forces surpass the critical value for tissue damage, they induce mechanical damage. Therefore, fruits can be damaged during handling, harvesting, transportation, and processing, and this damage has been described as a main source of decreased fruit quality, causing lower sale prices and losses to the grower or orchardist (Menesatti et al., 1999). Postharvest physical damage is common in tomatoes, even when the fruit is treated with care. Compared with other fruits, such as apples, tomatoes have lower storability, and they are susceptible to

\footnotetext{
Received for publication 13 Mar. 2018. Accepted for publication 7 July 2018.

This work was supported by the Korea Institute of Planning and Evaluation for Technology in Food, Agriculture, Forestry and Fisheries (IPET) through the Development of Transferring Equipment for Freshness Preservation of Paprika and Tomato in Greenhouse program, funded by the Ministry of Agriculture, Food and Rural Affairs (MAFRA) (315009033SB010).

${ }^{1}$ Corresponding author. E-mail: sicho@snu.ac.kr. This is an open access article distributed under the CC BY-NC-ND license (http://creativecommons. org/licenses/by-nc-nd/4.0/).
}

inappropriate conditions during harvesting, reloading, and transport (Abedi and Ahmadi, 2014; Tran et al., 2017).

Objective methods such as the use of data loggers and environmental factor sensors for monitoring the handling of vegetables and fruits are available. Monitoring the damage that occurs during the handling process is critical to understanding the causes of losses and to developing ways to overcome them. Information on the susceptibility of tomatoes to bruising is helpful for preventing and reducing bruise damage. Tomato bruises are not immediately visible, but they become apparent after handling and a certain amount of time on the shelf. Many studies have focused on the results of the mechanization of fruit grading and harvesting regarding different kinds of mechanical damage, and Allende et al. (2004) characterized factors that result in puncture damage during handling. Bruises develop in the flesh of the damaged fruit as an internal browning region resulting from the oxidation of phenolic compounds and tissue breakdown (Opara and Pathare, 2014). Currently, external bruising of tomatoes has not been clearly described in relation to internal bruising. Most researchers who have studied the influence of impact damage have studied externally visible damage instantly after harvesting or grading. These researchers often classify damage as visible permanent deformations (Sargent et al., 1989) or externally visible skin cracking and rupture (Geyer et al., 2002). Techniques to analyze damaged fruits with a nondestructive method have used thermal imaging devices (Cui et al., 2017a; Kheiralipour et al., 2013). Doosti-Irani et al. (2016) used a thermal imaging camera to assess the relationship between the deep internal temperatures of tested fruit and the external temperatures at the point where bruising appeared using thermal maps and thermal bruise depth. By multiple regression analysis, the results of analysis of variance showed that impact energy and impact region had a significant effect on temperature, which provided the basis of nondestructive fruit bruise detection. Despite the various types of damage studies, no such information is available on the combination of factors determining bruise susceptibility and actual impact-related bruise damage, and the assessment of drop-impact bruising associated with dropping tomatoes is lacking.

The bruise susceptibility of vegetables and fruits is a gauge for reactions to external forces and relies on some factors such as texture, variety, maturity, firmness, temperature, water status, shape, and size; as well as multiple internal factors, such as cell shape, cell wall strength, elasticity, and internal structure (Scheerlinck et al., 2006). Fruit texture is regarded as a primary contributing factor to bruise susceptibility and may differ significantly among fruits (Kerstens et al., 2000). Harker et al. (2010) performed a broad review of the different aspects of fruit texture and listed the factors that strongly affect fruit texture in general. The texture of tomatoes is basically reflected by the mechanical properties of pericarp tissue. Variations in the texture of a fruit change the mechanical properties of the fruit, thus influencing the bruise susceptibility of the fruit. An experiment involving a well-designed model of bruising is required to determine the factors that influence bruising susceptibility in tomato fruit. In the current study, impact force was monitored by an accelerometer at a farm. Then, bruising and changes in quality were observed by performing an experiment in the laboratory according to the analysis of the results from the farm. The current findings could help to determine threshold values for the extent of bruising that does not lead to further tissue damage and to evaluate of tomato bruising.

The objectives of the current study were to monitor the postharvest process at the farm and to develop multivariate analysis models using weight loss, firmness loss, and an assessment of bruising, together with fruit properties, impact force level, storage environment, and storage period as independent variables to determine the bruise susceptibility of red, ripe tomatoes.

\section{Materials and Methods}

Tomato farm experiment. To survey the fieldwork performed and select the corresponding impact force levels, the peak impact forces in a series of events during harvest and in scoring lines at a Korean tomato farm were determined from trials. Data loggers 
(BeanAir, Germany) were used to monitor acceleration, temperature, and humidity (BeanDevice; acceleration, $0-50 \mathrm{~g} \pm 10 \%$; temperature range, $-40{ }^{\circ} \mathrm{C}$ to $75^{\circ} \mathrm{C} \pm 0.2^{\circ} \mathrm{C}$; $\mathrm{RH}, 0 \%$ to $100 \% \pm 1.8 \%$ ). The acceleration sensors are wireless accelerometers dedicated to shock measurement with integrated data loggers. With a wakeup time of less than $10 \mathrm{~ms}$, these sensors can quickly track and monitor any type of shock event. In the experiment, the triaxial acceleration sensors were fitted at the bottom and side of the box to obtain three-axis (x, y, and z) impact data. The data loggers, which have a storage capacity of up to 1 million data points, were set to record real-time acceleration (a) at 1-s intervals.

Measurements of impact force yielded forces ranging from 0.2 to $4 \mathrm{~g}$ for harvesting, 4 to $8 \mathrm{~g}$ for cases during scoring, and more than $8 \mathrm{~g}$ for grading with worn or poorly adjusted and badly maintained equipment. The relationship between drop height (measured in centimeters) and impact force (measured in Newtons) recorded by the vibration meter (model ST-140D; Tenmars, Taiwan) was determined by dropping tomato samples weighing $250 \mathrm{~g}$ from heights of $20,30,40$, and $50 \mathrm{~cm}$. The vibration meter could measure velocity (range, $0.5-199.9 \mathrm{~m} \cdot \mathrm{s}^{-2}$; resolution, $0.1 \mathrm{~m} \cdot \mathrm{s}^{-2}$ ) and acceleration (range, 0.5-199.9 $\mathrm{mm} \cdot \mathrm{s}^{-1}$; resolution, $\left.0.1 \mathrm{~mm} \cdot \mathrm{s}^{-1}\right)$.

Table 1. Different classification impact levels applied to tomato fruit.

\begin{tabular}{lcc}
\hline Level & Impact force $(\mathrm{N})$ & Impact energy $(\mathrm{J})$ \\
\hline 1 & 2.45 & 0.490 \\
2 & 3.95 & 0.735 \\
3 & 5.68 & 0.980 \\
4 & 7.65 & 1.225 \\
\hline
\end{tabular}

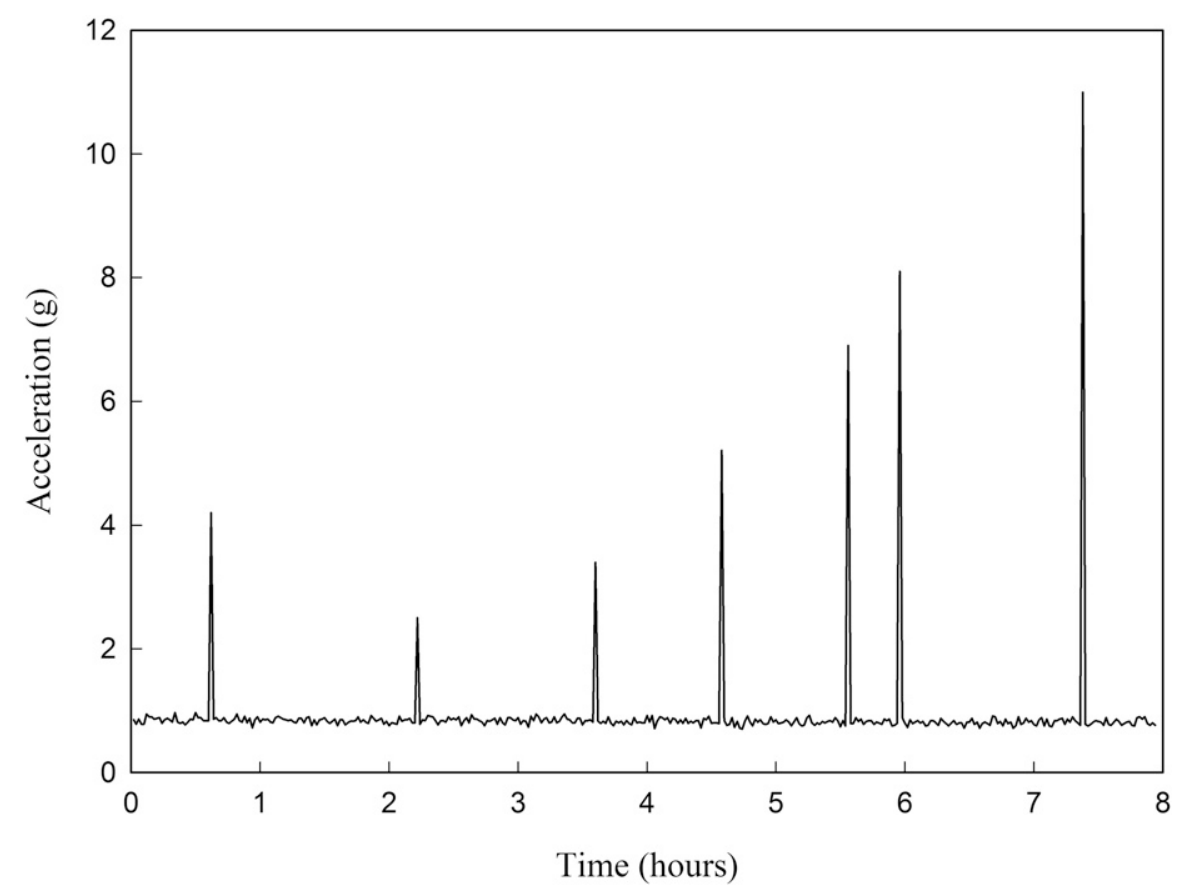

Fig. 1. Impact history for the postharvest processing of tomatoes at a farm.
The sensor was attached to one side of the tests on each side were repeated three times. Each tomato sample was examined on two sides.

Indoor experiment. The tested tomatoes ('Dotaerag Dia') were harvested in 2017 from Toechon $\left(37^{\circ} 45^{\prime} \mathrm{N}, 127^{\circ} 32^{\prime} \mathrm{E}, 43.75 \mathrm{~m}\right.$ a.s.l.), Gwangju-si, Gyeonggi-do, the Republic of Korea. The firmness of tomatoes is greater in spring. The color of the immature fruit is medium green; when ripe, the fruit is soft red. A total of 120 tomatoes were carefully handpicked at the red, ripe stage from several randomly selected branches to guarantee freshness and to prevent bruise damage during picking. The tomatoes were moved carefully to the laboratory, and the experiment was started immediately (May 2017). The tomato samples were separated into two groups and stored in two different environments during the measurements, and the longest storage period before disposal was $10 \mathrm{~d}$. The first group was stored at a controlled temperature of $10^{\circ} \mathrm{C}$ and an $\mathrm{RH}$ of $85 \%$, and the second sample group was stored at room temperature $\left(25^{\circ} \mathrm{C}\right)$ and at a lesser $\mathrm{RH}$ $(40 \%)$. In addition, in each storage environment, the tomatoes were separated into five groups, and the tomatoes assigned to each group were subjected to different drop tests. For each impact force level and temperature condition, 24 tomatoes were tested, and each tomato was subjected to impact once. Four groups were impacted at a force level of 1, 2, 3 , or 4 (one level per group), and the fifth group had no impact treatment, serving as the control group. Measurements of fruit quality, such as external color, firmness, and weight, were performed before and after the impact treatments. tomato before it was dropped, and drop
It was necessary to analyze and calculate the basic parameters of dropping impact theoretically to determine the dropping force and bruise susceptibility of tomatoes. Considering the dropping impact characteristics of fruit, a kinematic analysis may be performed by assuming that 1) samples are considered spheres, 2) air resistance was negligible and all the samples were subjected to the same impact force, and 3) the vibrations of the samples were negligible (Mohsenin et al., 1978). In Eqs. [1] through [3], the impact energy ( $W$, measured in joules), impact force $(F$, measured in joules), and acceleration (a; measure in Newtons per kilogram or meters per second) during contact were calculated as follows:

$$
\begin{aligned}
W=m \times g \times h & =\frac{1}{2} m \times v_{t}^{2}-\frac{1}{2} m \times v_{0}^{2} \\
F & =m \times a \\
a & =\frac{v_{t}-v_{0}}{t}
\end{aligned}
$$

where $m$ is sample mass, $g$ is acceleration resulting from gravity, $h$ is drop height, $v_{t}$ is the velocity of the sample at the last moment of dropping, $v_{0}$ is the velocity of the sample at the beginning of dropping, and $t$ is dropping time.

To deliver controlled impact forces to the tomatoes, we used an instrumented pendulum. A pendulum impactor is commonly used for impact tests (Opara and Pathare, 2014). The pendulum was comprised of a rotating wooden arm and a fixed metal structure. The pivot was at the upper end of the arm, and bearings were used at the pivot to minimize friction during the movement of the arm. The wooden arm had the impactor attached at the lower end, forming the pendulum. The impactor was a hemispherical piece of aluminum with a radius of $35 \mathrm{~mm}$ and a weight of $250 \mathrm{~g}$ to simulate best the shape of a tomato fruit. The impactor was attached at different points along the arm to apply different impact forces to the tomatoes.

Four classification impact levels were applied as outlined in Table 1. By adjusting the length of the arm, different impacts were applied to tomato samples. The impact force levels in the experiment were chosen above the critical impact level of tomatoes and the results obtained by Crisosto et al. (2001). The softest firmness at which a tomato did not develop bruising was defined as the critical bruising threshold. The critical bruising thresholds, when exposed to four different bruising potential levels, were calculated by using the $99 \%$ confidence limit of the relationship between bruising versus firmness at a given bruising potential level of impact. The lower limit of the applied impact level was based on the measured acceleration during transportation on a flat surface, but the greater impact level was based on mechanical size classification and packing. The least impact level was close to the detection 
limit of bruise damage. Levels 3 and 4 represented bruises clearly and were easily detectable after a period of storage.

The texture of vegetables and fruits can be evaluated by market experts or by a sensory panel. Each tomato sample was labeled, weighed, and subjected to color and firmness tests. Tomato weight was measured using a scale (model CU/XB 4200HW; CAS, Republic of Korea). Surface color and firmness can be established with a series of different nondestructive or destructive measurement tools. External firmness was measured objectively using a nondestructive firmness tester (model HPE 11 Fff; Bareiss, Oberdischingen, Germany). The firmness tester gauged the firmness of the tomato on the basis of the force-distortion reaction, with distortion of $1 \mathrm{~mm}$. The firmness value was Fff, which means fruit pulp firmness, and the measurement was nondestructive. After calibration with a white-plate standard [ $L$ (lightness) $=$ 97.26, $a$ (green-red color component) $=0.08$, and $b$ (blue-yellow color component) $=1.81$, a CR-300 chroma meter (Minolta, Osaka, Japan) was used (C illuminant, $0^{\circ}$ viewing) to detect the surface color of the tomato samples. The values of the coordinates $L$, $a$, and $b$, and the firmness of each sample were recorded as the mean of three values at the different midpoint locations of each sample. Color indices such as hue angle $\left(\mathrm{h}^{\circ}\right)$ (Eq. [4]) and chroma (C) (Eq. [5]) have been suggested as more realistic measures of color from a human sensorial

Table 2. Mean occurrence of impacts for tomatoes at the farm.

\begin{tabular}{lcc}
\hline Impact level $(\mathrm{g})$ & No. of impacts & Occurrence $(\%)$ \\
\hline $0.2-1.9$ & $378 \pm 15.3$ & 97.4 \\
$2-3.9$ & $3 \pm 0.2$ & 0.8 \\
$4-5.9$ & $2 \pm 0.2$ & 0.5 \\
$6-7.9$ & $2 \pm 0.2$ & 0.5 \\
$8-12$ & $2 \pm 0.2$ & 0.5 \\
$12+$ & $1 \pm 0.1$ & 0.3 \\
\hline
\end{tabular}

Values represent the mean \pm SD of 10 harvest boxes. point of view (McGuire, 1992), and both parameters were calculated with the following equations. In addition, $\Delta \mathrm{E}$ (Eq. [6]) was used to analyze the overall color change of $L, a$, and $b$.

$$
\begin{gathered}
\mathrm{h}^{\circ}=\arctan \left(\frac{b_{t}}{a_{t}}\right) \\
\mathrm{C}=\sqrt{\left(a_{t}^{2}+b_{t}^{2}\right)} \\
\Delta \mathrm{E}=\sqrt{\left(a_{t}-a_{0}\right)^{2}+\left(b_{t}-b_{0}\right)^{2}+\left(L_{t}-L_{0}\right)^{2}}
\end{gathered}
$$

where $\mathrm{h}^{\circ}$ is defined from a color wheel, with red-purple at an angle of $0^{\circ}$, yellow at $90^{\circ}$, blue-green at $180^{\circ}$, and blue at $270^{\circ} ; \mathrm{C}$ is the color saturation of the samples, which changed from a dull (low value) to a vivid color (high value) (Topuz et al., 2009); In Eq. [6], E represents the color difference. $L_{0}, a_{0}$, and $b_{0}$ are the original external colors at the beginning of the experiment, and $L_{t}, a_{t}$, and $b_{t}$ are the colors measured during storage.

The dependent variables were weight loss, firmness loss, and whether the dropimpact force resulted in bruising. These variables were used to determine the bruise model. The dependent variables were measured $0,2,4,6,8$, and $10 \mathrm{~d}$ after impact treatment, and weight loss (Eq. [7]) and firmness loss (Eq. [8]) were determined based on the following equations:

$$
\begin{aligned}
& \text { Weight loss }(\%)=\frac{W_{0}-W_{t}}{W_{0}} \times 100 \\
& \text { Firmness loss }(\%)=\frac{F_{0}-F_{t}}{F_{0}} \times 100
\end{aligned}
$$

where $W_{0}$ is the original weight at the beginning of the experiment (measured in grams), $W_{t}$ is the fruit weight during storage (measured in grams), $F_{0}$ is the original firmness at the beginning of the experiment

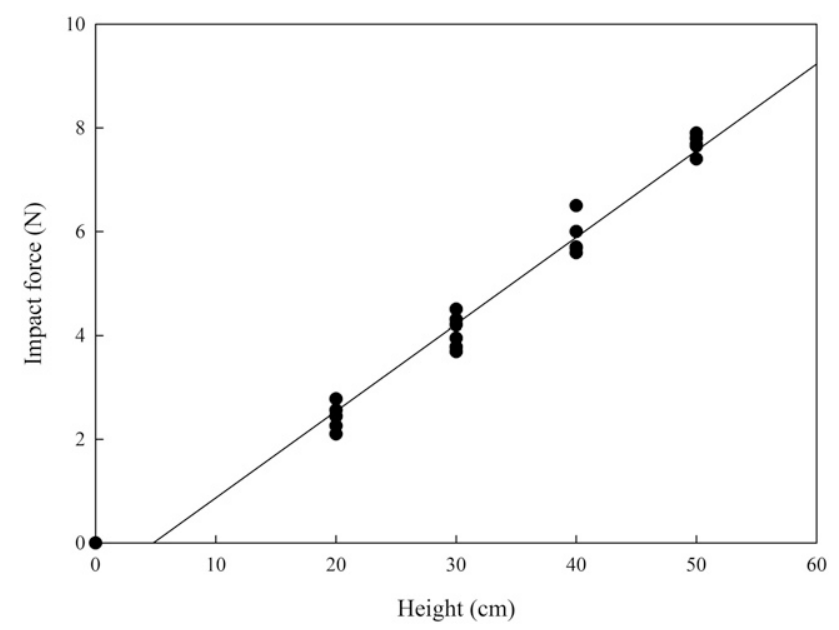

Fig. 2. Correlation between impact force (measured in Newtons) and drop height (measured in centimeters). The solid line indicates the result of linear regression.

(measured in units), and $F_{\mathrm{t}}$ is the fruit firmness during storage (measured in units).

Because the bruises were not detectable immediately after impact treatments, bruising was assessed after $2 \mathrm{~d}$, within $10 \mathrm{~d}$ of storage, as were the other variables. Fruit bruises were evaluated using a binary response variable to evaluate the bruise as absent (0) or present (1). When no local bruising of the external tissue was noticed, the bruise parameter was set to 0 . When the impacted area seemed to be damaged and soft, the bruising parameter was set to 1 . The level of bruising was not considered. The tomatoes in the control set, which were not impacted, were all categorized as 0 , and the tomatoes in treatment groups were divided into groups categorized as either 0 or 1 , depending on whether they were damaged.

Model development using multivariate analysis. For the linear multiple regression analysis, we used two kinds of linear regression models. The independent variables of the first model were impact level, weight, storage day, environment, and external color, such as $L, a, b, \mathrm{C}$, and $\Delta \mathrm{E}$, and the dependent variable was weight loss. The second linear regression model was similar to the first one, except that the dependent variable was firmness loss and the independent variables were weight, storage day, environment, firmness, and external color variables, such as $a, b, \Delta \mathrm{E}$, and $h^{\circ}$. A backward stepwise multiple regression method was used to select the relevant independent variables related to the dependent variables at $P<0.05$. Moreover, a chi-square test was implemented based on experimental and predicted data to verify the accuracy of the multiple regression models.

In addition, ANN-based regression models, which could determine weight loss and firmness loss rates by using fruit-quality factors obtained from tomato samples, were developed. The models consisted fundamentally of the following parts: input, hidden, and output layers. Also, both a supervised learning method and Levenberg-Marquardt backpropagation algorithms were used. For the development of the ANN-based models, all the factors of fruit quality, environment, and impact force levels were set as the input (independent variables), and both weight loss and firmness loss values were set as the output (dependent variables). The node number of the hidden layer was set as 3 . In addition, a sigmoid function was used as the activation function, and the iteration number was limited to a maximum of 1000 to prevent overflow during calculations.

Logistic regression is an appropriate statistical method for analyzing a binomial response and binary data. Logistic regression is a specific example of a generalized linear regression model in which the dependent variable or the observed response variable is first transformed to logit values, and a linear relationship with a set of interpreted variables is then assessed. Eq. [9] shows this relationship (Hosmer et al., 2013). 


\begin{tabular}{|c|c|c|c|c|c|c|}
\hline \multirow[b]{2}{*}{ Model } & \multirow[b]{2}{*}{ Equation } & \multirow[b]{2}{*}{ Adjusted $R^{2}$} & \multirow[b]{2}{*}{ Chi-square test } & \multicolumn{3}{|c|}{ Residuals } \\
\hline & & & & Minimum & Maximum & Mean \\
\hline 2 & $\begin{aligned} \mathrm{FL} & =25.505+1.978 \cdot \mathrm{SD}-15.919 \\
& \cdot \mathrm{Ev}-0.028 \cdot \mathrm{W}-1.093 \\
& \cdot \mathrm{F}+1.385 \cdot \mathrm{a}-3.627 \cdot \mathrm{b}+0.757 \\
& \cdot \mathrm{E}+95.029 \cdot \mathrm{H}\end{aligned}$ & 0.94 & 27 & -29.06 & 13.37 & 5.17 \\
\hline
\end{tabular}

$\mathrm{WL}=$ weight loss; $\mathrm{SD}=$ storage day; $\mathrm{DL}=$ drop level; $\mathrm{Ev}=$ environment; $\mathrm{W}=$ weight $\mathrm{L}=$ lightness; $\mathrm{a}=$ redness; $\mathrm{b}=$ yellowness; $\mathrm{E}=\Delta \mathrm{E}(\mathrm{color}$ difference); $\mathrm{C}=$ chroma; $\mathrm{FL}=$ firmness loss; $\mathrm{F}=$ firmness; $\mathrm{H}=$ hue.

$$
\operatorname{logit}=\log \left(\frac{p}{1-p}\right)=\alpha+\sum_{k=1}^{m} \beta_{k} x_{i k}
$$

where $p$ is the probability of an event $[0,1]$ and is defined by the proportion of bruised tomatoes in the current analysis. A set of $m$ interpreted variables was determined by the logit value; $\alpha$ was intercept parameter; $\beta_{k}$ was determined by the $\mathrm{k}_{\text {th }}$ interpreted variable and identified its significance; $x_{i k}$ was explanatory variable; and $\beta_{k} x_{i k}$ was the regression coefficient multiplied by some value of the predictor. $i$ represented the indicator that indicated whether it had been impacted. Probabilities were calculated from the logistic regression function (Eq. [9]). All continuous covariates were standardized before the analysis $[N(0,1)]$.

De Ketelaere et al. (2006) proposed the analysis just described to link loading conditions to the resulting bruise damage. In addition, this method is appropriate for assessing the internal and external factors that influence the susceptibility of tomatoes to bruising when using a binary response variable to assess bruise damage. Stepwise logistic regression was carried out on the full dataset to select an appropriate subset of independent variables. According to the Akaike information criterion (AIC), which is an estimator of the relative quality of statistical models, where the preferred model or independent variable is the one with the minimum AIC value, variables were substituted in the model or deleted from the model (linear and logistic). All important and significant independent variables were included automatically.

All statistical analyses, development of algorithms needed for analysis, and multivariate analysis model construction were performed using R 3.4.2 (R Development Core Team; Boston, MA). The output data were plotted using SigmaPlot, version 12.5 (Systat Software Inc., San Jose, CA).

\section{Results}

Studies at a tomato farm. Tomatoes to be sold in the Republic of Korea were handharvested into plastic boxes at the farm and moved to a sorting facility by forklifts to flatbed trucks. At the sorting facility, fresh tomatoes were also moved by forklifts, tipped artificially into a tomato screening machine, moved up a conveyer belt, and dropped from the belt into thick paper boxes. The total
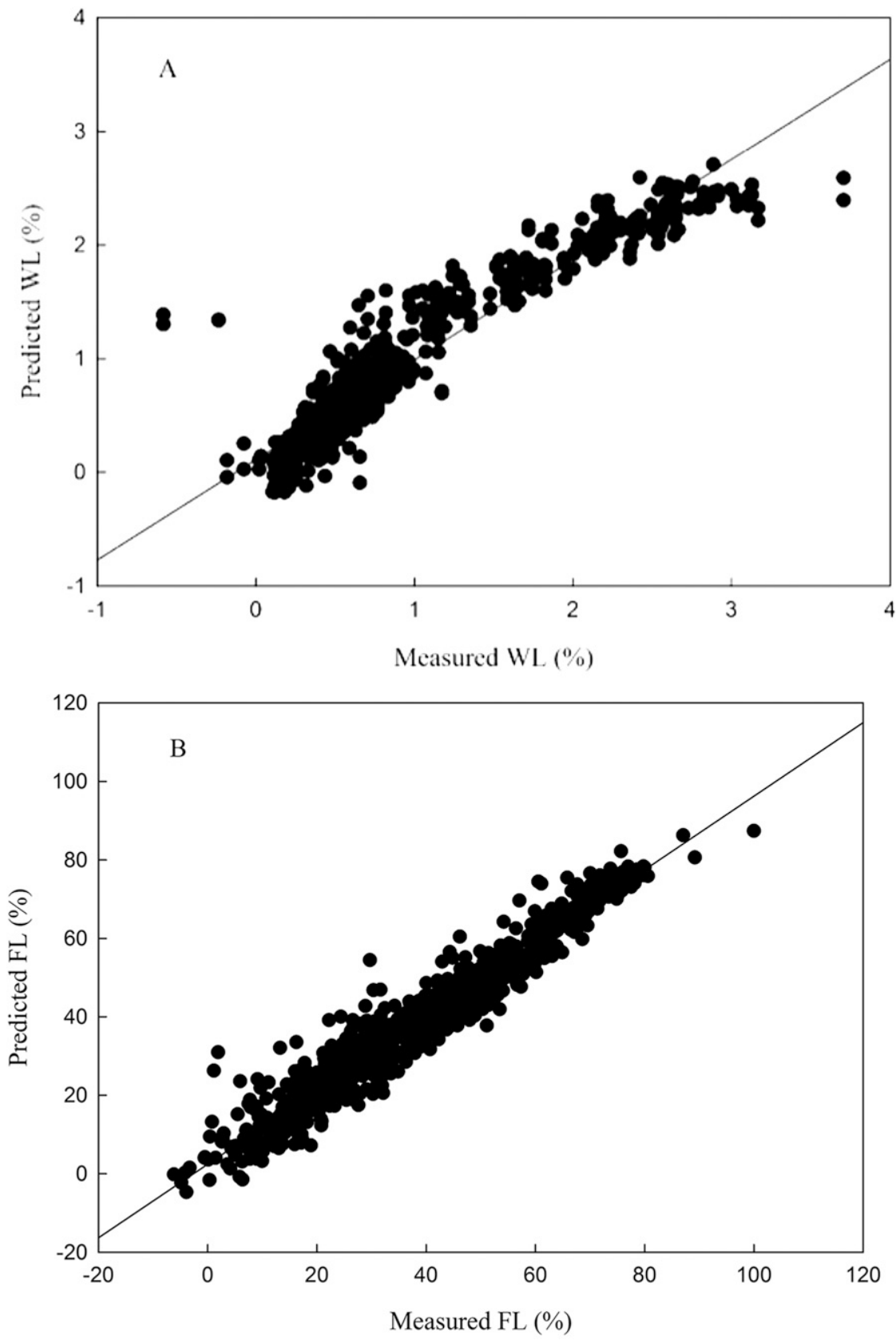

Fig. 3. Results for the prediction of weight loss (WL) and firmness loss (FL) values based on linear regression models. (A) Weight loss. (B) Firmness loss. The solid line indicates the result of linear regression.

process varied in duration between $6 \mathrm{~h}$ and $12 \mathrm{~h}$ (mean, $8 \mathrm{~h}$ ). The data loggers provided an efficient method for measuring the handling of fruit during the postharvest process.
The impact history of tomatoes transported to the sorting facility at the farm is shown in Fig. 1. For postharvest processing and transport, large impact forces were recorded 
during unloading and dumping, and when the boxes were moved along a nonflat floor. A summary of the impact forces by category (0.2-1.9 g, 2-3.9 g, 4-5.9 g, 6-7.9 g, 8-11.9 $\mathrm{g}$, and $>12 \mathrm{~g}$ ) is shown in Table 2 . The majority of impacts $(97.4 \%)$ were less than $2 \mathrm{~g}$. The larger impacts ( $>8 \mathrm{~g}$; equivalent to a drop height of $\approx 50 \mathrm{~cm}$ or more) generally occurred during dumping and passing over the nonflat floor. In Fig. 1, the acceleration peak was placed in the harvesting stage before $2 \mathrm{~h}$. From 2 to $6 \mathrm{~h}$, the acceleration peak was generated at the stage of transfer from the harvesting site to the classification site. After $6 \mathrm{~h}$, the highest acceleration peak occurred at the stage of classification, such as dumping and slipping.

The relationship between the impact force (measured in gravitational acceleration $\mathrm{m} / \mathrm{s}^{2}$ ) recorded by data loggers and the height that a sample was dropped is shown in Fig. 2. The curves show that the impact force increased with drop height $\left(R^{2}=0.926\right)$, and this was similar to the data logger results.

The results of the models developed using multivariate analysis. Table 3 shows both of the final linear regression models and the backward-selected independent variables. For these models, the plot of predicted values against measured values is shown in Fig. 3A and B. A good fit was found for the results. As shown in Table 3, the model of firmness loss was more predictive than the model of weight loss, and the adjusted coefficient of determination for firmness loss was 0.93, which was greater than the coefficient for weight loss (0.88). This result indicated there was a systematic relation between predicted and measured values. In addition, most of the results ranged from $0 \%$ to $40 \%$, except for outliers with large deviations. The causes of these outliers were analyzed as follows: the occurrence of an artificial measurement error in the experiment and the influence of the process of ripening in the experiment, because since tomato is a postripeness fruit.

Figure 4 shows the evaluation performances of the ANN models for the weight loss and firmness loss rates of tomato samples. The performance of each model was assessed using the coefficient of determination. Based on the test results of the ANN models, the performance of the model considering the weight loss rate $\left(R^{2}=0.95\right)$ was slightly better than that considering the firmness loss rate $\left(R^{2}=0.91\right)$. Figure 4 shows the most strongly correlated ANN models for the values of weight loss and firmness loss. In the ANN analysis structure, the hidden layer node number, the decay rate, and the number of iterations were 3, 0.1 and 1000 , respectively. Using the same settings, the ANN prediction models were applied to firmness loss and weight loss rates, and the results showed that the model incorporating predictions of weight loss rates was better than the model incorporating predictions of firmness loss rates.

Logistic regression analyses were used to assess the experimental data. Table 4 summarizes the model with drop impact force,
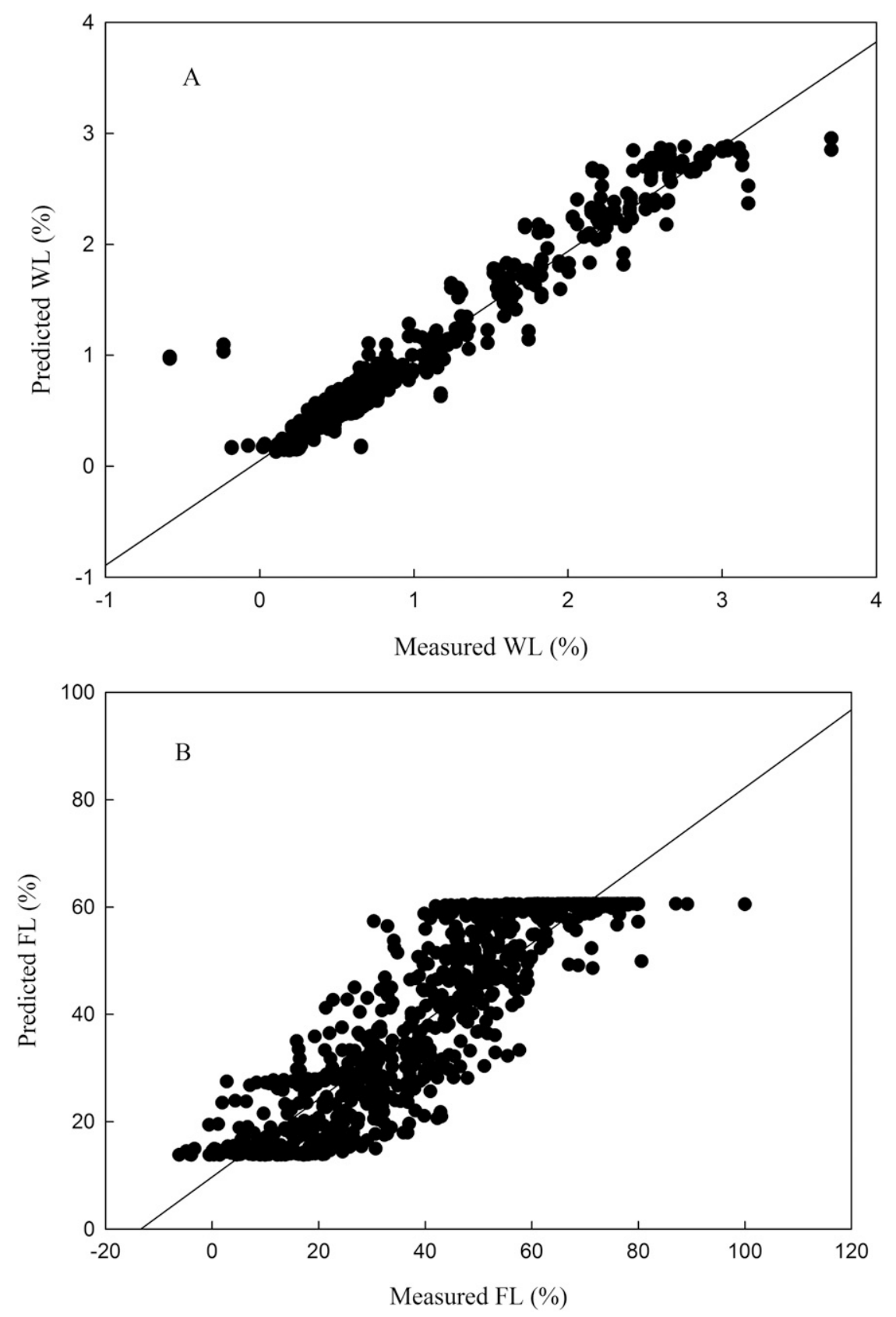

Fig. 4. Results for the prediction of weight loss (WL) and firmness loss (FL) values based on artificial neural network models. (A) Weight loss. (B) Firmness loss. The solid line indicates the result of linear regression.

Table 4. Summary of the logistic regression analysis of factors affecting the presence of bruising in tomato fruits.

\begin{tabular}{|c|c|c|c|c|}
\hline $\begin{array}{l}\text { Explanatory independent } \\
\text { variable }\end{array}$ & $\begin{array}{c}\text { Parameter } \\
\text { estimate }(\beta)\end{array}$ & $\mathrm{AIC}^{\mathrm{z}}$ & $\begin{array}{l}\text { Effect Wald } \\
\text { chi square }\end{array}$ & $\begin{array}{c}\text { Adjusted } \\
R^{2}\end{array}$ \\
\hline Intercept & 8.41 & 372.52 & 5.34 & 0.42 \\
\hline Hue & 11.52 & 372.66 & 4.36 & \\
\hline$a^{y}$ & 1.93 & 376.27 & 17.11 & \\
\hline$\Delta \mathrm{E}$ & 0.24 & 377.48 & 24.06 & \\
\hline Chroma & -2.15 & 379.12 & 9.68 & \\
\hline Storage day & -0.52 & 400.27 & 7.22 & \\
\hline Environment & 4.21 & 404.20 & 9.76 & \\
\hline Firmness & -0.17 & 409.60 & 4.17 & \\
\hline
\end{tabular}

${ }^{\mathrm{z}}$ The AIC is 354.52 for the intercept only; 372.52 is for the intercept and covariates. $\mathrm{AIC}=$ Akaike information criterion.

storage environment, storage period, and related fruit-quality parameters. The results of the model with the lowest AIC value are shown, which is the preferred model. The influence of the impact force and fruit-quality parameters appeared to be well balanced on the basis of chi-square values. In general, the flesh and external skin color, storage period, 
and firmness were closely related to the presence of bruising. The focus was on model fit in assessing the logistic model. Figure 5 shows the standardized residual deviances of the logistic model. Absolute values greater than 1.5 were poorly accounted for, although this result does not necessarily mean that these values are outliers. Particularly considering the limitations of the experimental design, such models could not illustrate conditions that occur in real life. For example, fruit that was impacted with a low level of force could appear to be bruised. In such cases, the model predicted no bruise damage, which did not agree with observations. Nonetheless, these data were retained in the dataset because they show the weak points of the model and were used to illustrate the large degree of biologic variation that is usually present.

Figure 6 shows the last logistic regression model for tomatoes with several levels of impact. The vertical bars indicate the bruise prediction values for each given condition. The coordinates along the horizontal plane of the graph indicate storage period and firmness. These two factors were chosen on the basis of the AIC results in Table 4. As shown in Fig. 6, a greater firmness value and shorter storage period predicted a lower probability of impact bruising. Alternatively, if the firmness value was low and the storage period was long, the probability of impact bruising increased. In short, the logistic model is relevant to susceptibility trials, and it could be carried out with additional extrinsic and intrinsic properties of fruits to determine the factors that influence tomato bruising.

Temperature had a positive effect on the weight loss and firmness loss rates (Fig. 7); a warmer temperature caused more bruising. However, RH showed the opposite results. The largest difference between these two storage environments was observed at lower impact force levels. This difference ranged from $\approx 62 \%$ for the control set tomatoes to $45 \%$ for greatest impact force.

\section{Discussion}

The results of the tests introduced here and the application of accelerometer-based measurements can be used to supply information regarding the hazards of mechanical damage (particularly in bruising) during postharvest processes such as handling, storage, and transport. Then, bruising models were described by a combination of impact force level and tomato properties. The impact force level and some of the interactions with tomato properties have a substantial effect on the bruising damage of a tomato. When tomatoes were impacted at a high impact force level, the probability to develop a bruise was greatly increased because the high impact force results in high peak contact stress.

Fruit parameters. The influence of the external color on fruit bruising is important

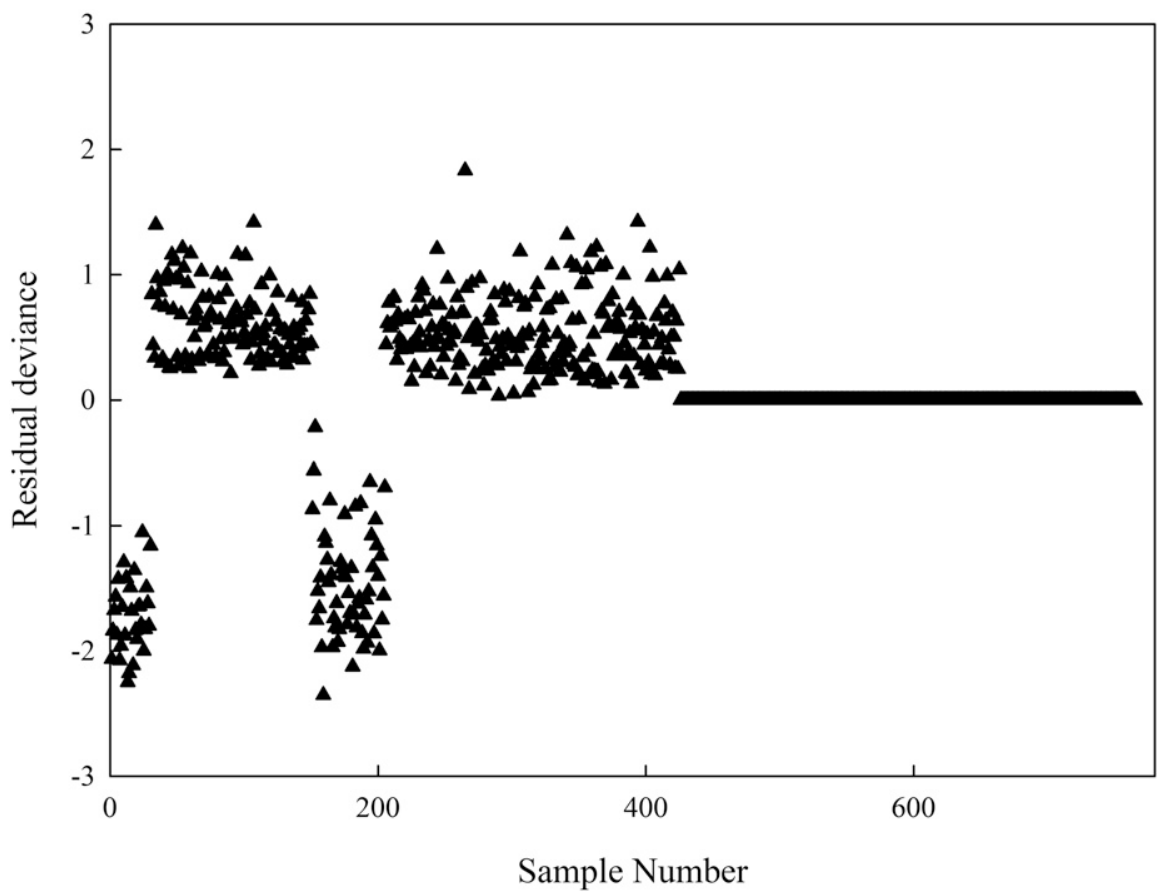

Fig. 5. Residual deviances for the logistic model containing impact force and fruit-quality parameters Residual deviances with an absolute value of more than 1.5 are poorly accounted for by the model.

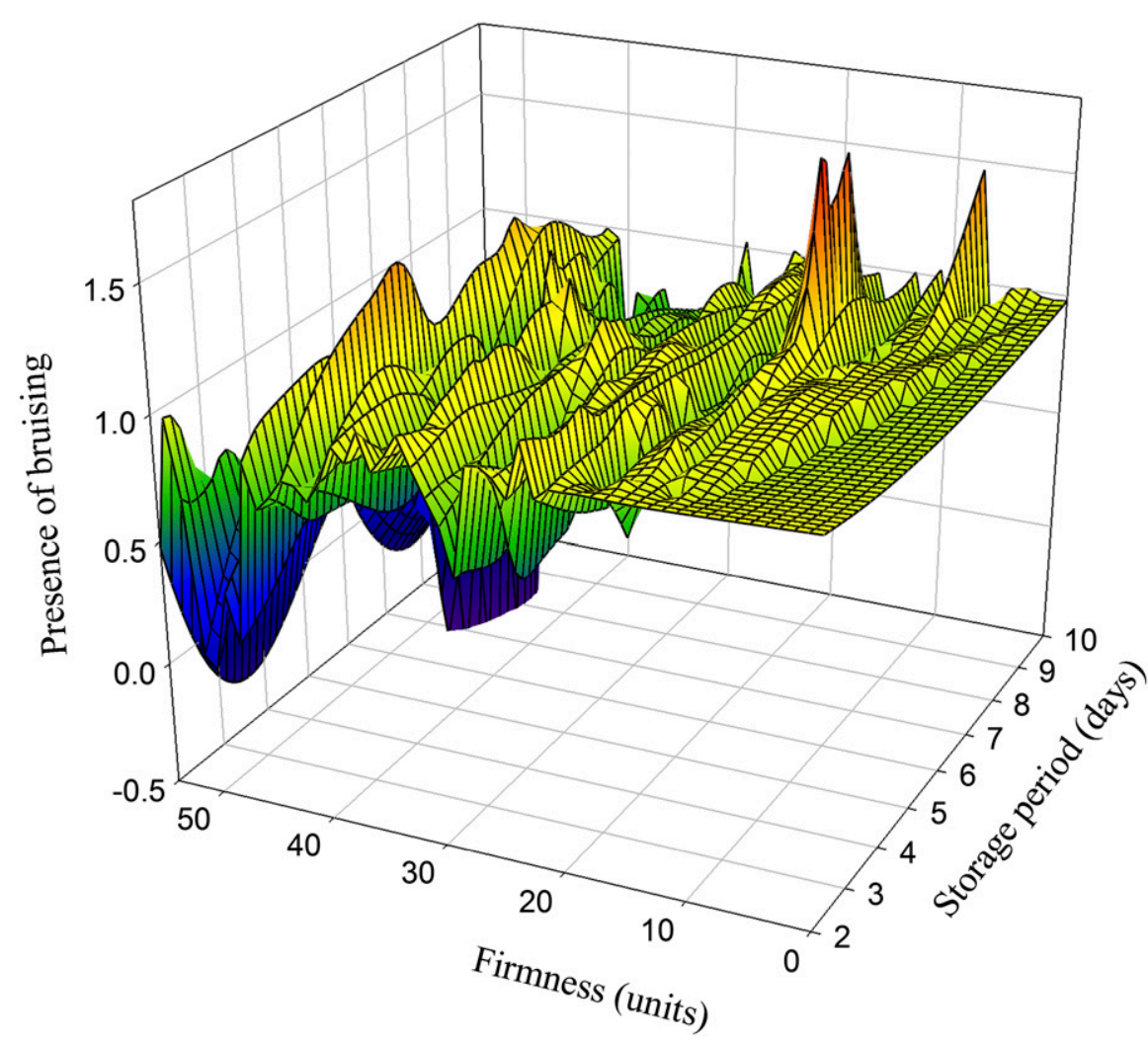

Fig. 6. The probability of developing a bruise based on the logistic model for tomato fruits.

but difficult to explain. In addition, mass has a dual influence because heavier, larger fruit have less cell-to-cell contact and lower concentrations of cell wall material, which causes decreased tissue strength (Harker et al., 2010). In our study, the effect of the firmness on bruising was high at the lowimpact force level and with the short storage period. Stiffer tomatoes were less susceptible to bruise damage at the low-impact force. This finding could be a result of the dominant effect of greater firmness. However, at the 

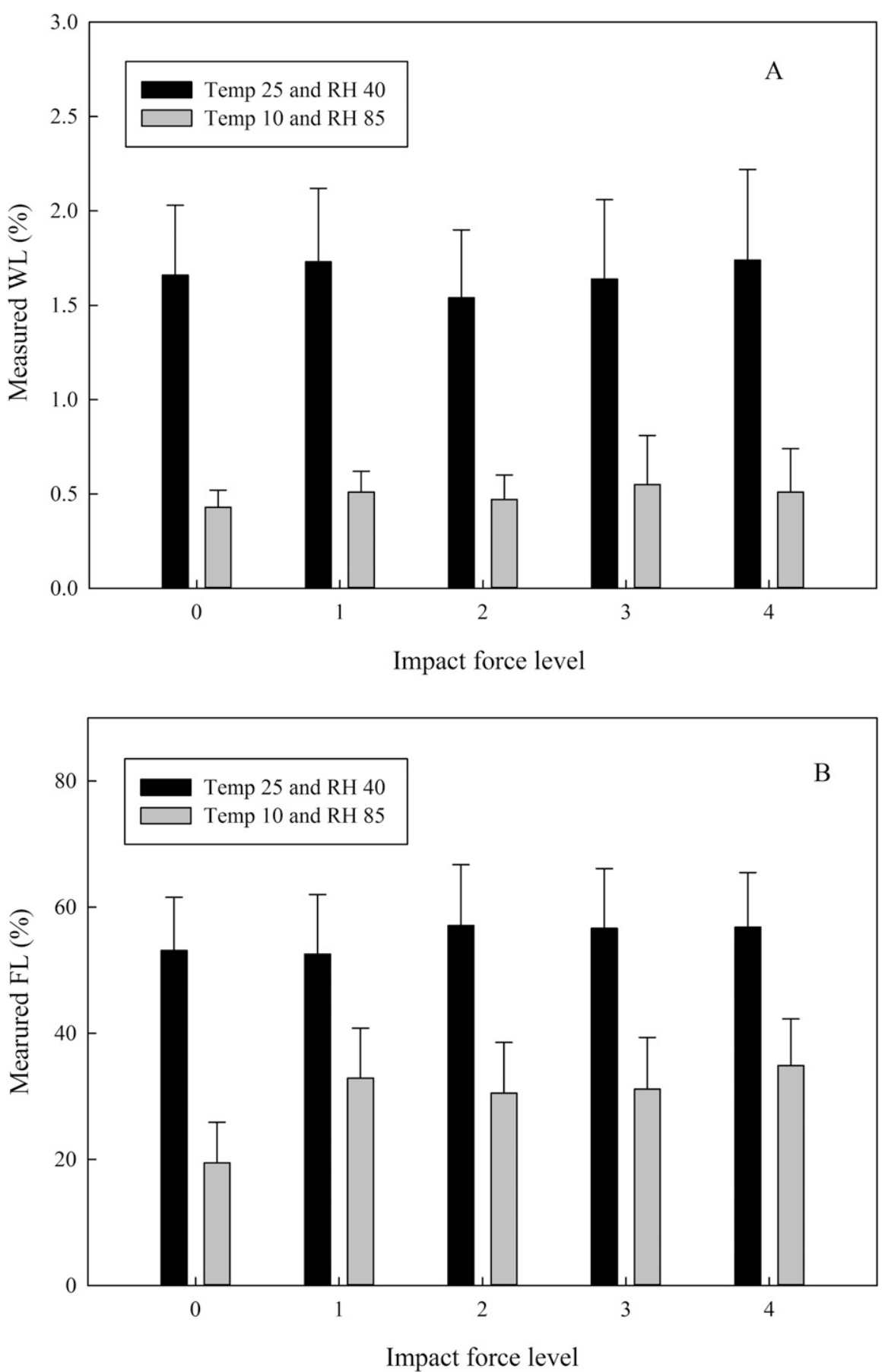

Fig. 7. Effect of storage environment on the weight loss (WL) and firmness loss (FL) rates of tomato fruit for each impact force level. (A) Weight loss. (B) Firmness loss. Values represent the mean \pm SD of each tomato group. Temp $=$ temperature (measured in degrees Celsius), $\mathrm{RH}=$ relative humidity (measured as a percentage).

high-impact force level, the greater peak contact force had a more dominant role than greater tomato firmness. Compared with less firm tomatoes, firmer tomatoes develop greater peak contact force when dropped from the same height. Studman (1997) stated that a decrease in cell wall integrity increases the susceptibility of tomatoes to bruising. The cells of mature fruit are less capable of withstanding external loading and can be damaged more easily. Van Linden and De Baerdemaeker (2004) showed that the mechanism of bruising is a combination of physical maturity. Therefore, overmature and soft tomatoes could be overly sensitive to bruise damage. As with many other kinds of fruits, firmer tomatoes could better withstand handling at harvest and thus have a longer storage potential (Cui et al., 2017b).

Environmental parameters. Temperature and RH also have a very important function in determining the postharvest life span of agricultural goods. In this experiment, tomatoes were stored in two different storage environments, including high-RH/low-temperature storage and low-RH/room-temperature storage. The degree of tomato bruising at room temperature was more severe than that at $10{ }^{\circ} \mathrm{C}$. In both storage conditions, the firmness loss rate and weight loss rate increased with the increase in the impact force level. In general, warm temperature and low $\mathrm{RH}$ had a negative effect on the likelihood of impact bruising, with warmer temperatures associated with more bruising. On the one hand, the metabolic rate is reduced with an increase in storage temperature, as is polygalacturonase activity, which increases bruise susceptibility at greater concentrations (Chun and Huber, 1998). On the other hand, cell walls become less flexible at colder temperatures (Studman and Boyd, 1994), which could cause deep bruising. Cold temperatures also contribute to changes in metabolic activity and fruit quality. In the current study, colder temperatures decreased bruising damage. Baritelle et al. (2000) stated that the influences of turgor and temperature on strain and stress failure, tissue toughness, shock wave speed, and the secant elastic modulus were extremely significant. After damage, if the fruit is kept at a warmer temperature, the bruise goes deeper. For this reason, fruit should be cooled quickly after packing.

Multivariate analysis evaluation. The use of multivariate models can determine objectively the parameters associated with the incidence of bruising under different impact force levels, and the use of better models can help better prevent bruise damage in practice. The linear regression models showed that susceptible tomatoes were subjected to a long storage period, high-impact force level, and warmer temperature. The presented bruise prediction logistic model includes firmness and storage environment as the main independent variables. The advantage of this model is that it can judge the possibility of tomato bruising through simple measurements (nondestructive firmness and color test) and variable inputs. The disadvantage of using this model is that the result is most likely influenced by special tomato characteristics (e.g., excessively hard pericarp tissue, overripeness). The contribution of the storage environment and the mechanical strength of the cell wall to firmness might change depending on the type of fruit and its physiologic conditions.

Although assessments of several internal and external parameters related to fruit can assist in predicting impact bruise damage, the $R^{2}$ statistics of the model fitting indicated that most of the variance is left unaccounted for. Particularly, predicting bruising at low-impact 
force levels and in tomatoes at the early stage of maturity is difficult. Some of this difficulty might be the result of the reactions of fruits at the molecular and cellular levels.

\section{Conclusions}

The main reasons for a reduction in tomato quality during transport and handling have been identified. The effects of handling were most severe during dumping and when tomatoes were dropped from a certain distance. Experimental results at a farm indicated that impacts during rough handling could exceed $12 \mathrm{~g}$, which is equivalent to a drop of $50 \mathrm{~cm}$ or more. The accurate and diverse data obtained by the pendulum tests and measurement devices could provide a basis for the detailed analysis of tomato bruise susceptibility. The linear regression and ANN models were effective in predicting the weight loss and firmness loss rates. A logistic regression demonstrated a link between the consequences bruise damage and the impact force delivered to the tomatoes. Tissue discoloration reflects damage to all kinds of fruit. Although membrane damage may follow greater impacts, the injured tissue did not significantly change in color, except for some color indices such as $\Delta \mathrm{E}, \mathrm{C}$, and $\mathrm{h}^{\circ}$. In contrast, in the most severe cases, water built up under the surface or in the following 2-3 $d$ and the affected tissue softened steadily. In addition, local changes in firmness were detected. Our methods had some benefit in reducing time-consuming repetitive experiments.

In summary, the damage evaluation and multivariate analysis methods proposed in this article can be used to determine the bruise susceptibility of tomatoes. Additional multivariate models may be helpful for studying the effect of different fruit handling processes on the risk of developing bruises after mechanical impact. This article provides a macroscopic analysis of tomato bruising in relation to structural aspects. The underlying molecular and cellular processes are not considered here, but are worth examining in future studies.

\section{Literature Cited}

Abedi, G. and E. Ahmadi. 2014. Bruise susceptibilities of Golden Delicious apples as affected by mechanical impact and fruit properties. J. Agr. Sci. 152:439-447.

Allende, A., M. Desmet, E. Vanstreels, B.E. Verlinden, and B.M. Nicolaï. 2004. Micromechanical and geometrical properties of tomato skin related to differences in puncture injury susceptibility. Postharvest Biol. Technol. 34:131-141.

Baritelle, A., G. Hyde, and J. Varith. 2000. Turgor and temperature effects on pear tissue failure. Proc. ASAE Annu. Milwaukee, WI, 9-12 July 2000.

Chun, J.-P. and D.J. Huber. 1998. Polygalacturonasemediated solubilization and depolymerization of pectic polymers in tomato fruit cell walls: Regulation by $\mathrm{pH}$ and ionic conditions. Plant Physiol. 117:1293-1299.

Crisosto, C.H., D. Slaughter, D. Garner, and J. Boyd. 2001. Stone fruit critical bruising thresholds. J. Amer. Pomol. Soc. 55:76-81.

Cui, J., M. Yang, D. Son, and S.-I. Cho. 2017a. Machine vision and thermographic imaging for determining of grading of tomato on postharvest. Proc. Trans. ASABE 10:1-12.

Cui, J., A. Yu, M. Yang, and S.-I. Cho. 2017b. Prediction of weight loss of low temperature storage tomato (Tiwai 250) by non-destructive firmness measurement. Korean J. Food Preserv. 24:181-186.

De Ketelaere, B., M. Desmet, and J. De Baerdemaeker. 2006. Determination of bruise susceptibility of tomato fruit by means of an instrumented pendulum. Postharvest Biol. Technol. 40:7-14.

Doosti-Irani, O., M.R. Golzarian, M.H. Aghkhani, H. Sadrnia, and M. Doosti-Irani. 2016. Development of multiple regression model to estimate the apple's bruise depth using thermal maps. Postharvest Biol. Technol. 116:75-79.

Geyer, M., B. Herold, B. Oberbarnscheidt, F. Jakovác, and B. Borsa. 2002. Minimized losses by mechanical tomato harvest in consideration of maturity and susceptibility. Proc. Agr. Eng. 1:225-226.

Harker, F.R., R.J. Redgwell, I.C. Hallett, S.H. Murray, and G. Carter. 2010. Texture of fresh fruit. Hort. Rev. 20:156-182.

Hertog, M.L., R. Ben-Arie, E. Róth, and B.M. Nicolai. 2004. Humidity and temperature effects on invasive and non-invasive firmness measures. Postharvest Biol. Technol. 33:79-91.

Hosmer, D.W., Jr., S. Lemeshow, and R.X. Sturdivant. 2013. Applied logistic regression. 3rd ed. Wiley, New York, NY.
Kerstens, S., W. Decraemer, and J. Verbelen. 2000. Viscoelastic properties of cell walls are related to the orientation of cellulose fibrils. Proc. Plant Bio. 27:487.

Kheiralipour, K., H. Ahmadi, A. Rajabipour, S. Rafiee, M. Javan-Nikkhah, and D. Jayas. 2013. Development of a new threshold based classification model for analyzing thermal imaging data to detect fungal infection of pistachio kernel. Agr. Res. 2:127-131.

Lubes, G. and M. Goodarzi. 2017. Analysis of volatile compounds by advanced analytical techniques and multivariate chemometrics. Chem. Rev. 117:6399-6422.

McGuire, R.G. 1992. Reporting of objective color measurements. HortScience 27:1254-1255.

Menesatti, P., C. Beni, G. Paglia, S. Marcelli, and S. D’Andrea. 1999. Predictive statistical model for the analysis of drop impact damage on peach. J. Agr. Eng. Res. 73:275-282.

Mohsenin, N., V. Jindal, and A. Manor. 1978 Mechanics of impact of a falling fruit on a cushioned surface. Trans. ASABE 21:5940600 .

Opara, U.L. and P.B. Pathare. 2014. Bruise damage measurement and analysis of fresh horticultural produce: A review. Postharvest Biol. Technol. 91:9-24.

Sargent, S., J. Brecht, and J. Zoellner. 1989. Assessment of mechanical damage in tomato packing lines. Proc. Trans. ASABE paper no. 89-6060.

Scheerlinck, N., M. Desmet, and J. De Baerdemaeker. 2006. Factors that affect tomato bruise development as a result of mechanical impact. Postharvest Biol. Technol. 42:260-270.

Studman, C. 1997. Factors affecting the bruise susceptibility of fruit. Proc. Conf. Plant 1:273-281.

Studman, C. and L. Boyd. 1994. Measurement of firmness in fruits and vegetables. AgEng. Conf. Milan report no. \#94-G-066.

Topuz, A., H. Feng, and M. Kushad. 2009. The effect of drying method and storage on color characteristics of paprika. Lebensm. Wiss. Technol. 42:1667-1673.

Tran, D.T., M.L. Hertog, T.L. Tran, N.T. Quyen, B. Van de Poel, C.I. Mata, and B.M. Nicolaï. 2017. Population modeling approach to optimize crop harvest strategy: The case of field tomato. Front. Plant Sci. 8:608.

Van Linden, V. and J. De Baerdemaeker. 2004. The phenomenon of tomato bruising: Where biomechanics and biochemistry meet. Acta Hort. 682:925-932. 